\title{
Evaluation of the Benefits of De-Escalation for Patients with Sepsis in the Emergency Intensive Care Unit
}

Takahiro Niimura ${ }^{1}$, Yoshito Zamami ${ }^{1,2}$, Toru Imai ${ }^{3}$, Kanako Nagao $^{4}$, Masafumi Kayano ${ }^{2}$, Hidenori Sagara ${ }^{5}$, Mitsuhiro Goda $^{2}$, Naoto Okada ${ }^{2}$, Masayuki Chuma ${ }^{6}$, Kenshi Takechi ${ }^{6}$, Masaki Imanishi ${ }^{2}$, Toshihiro Koyama ${ }^{7}$, Tadashi Koga ${ }^{8}$, Hironori Nakura ${ }^{4}$, Toshiaki Sendo ${ }^{9}$, Keisuke Ishizawa ${ }^{1,2}$

\begin{abstract}
${ }^{1}$ Department of Clinical Pharmacology and Therapeutics, Tokushima University Graduate School of Biomedical Sciences, Tokushima, Japan; ${ }^{2}$ Department of Pharmacy, Tokushima University Hospital, Tokushima, Japan; ${ }^{3}$ Department of Pharmacy, Nihon University Itabashi Hospital, Tokyo, Japan; ${ }^{4}$ Department of Emergency Pharmaceutics, Graduate School of Medicine Dentistry and Pharmaceutical Sciences, Okayama University, Okayama, Japan; ${ }^{5}$ Department of Pharmaceutical Information Sciences, Matsuyama University, Ehime, Japan; ${ }^{6}$ Clinical Trial Center for Developmental Therapeutics, Tokushima University Hospital, Tokushima, Japan; ${ }^{7}$ Department of Clinical Pharmacy, Graduate School of Medicine, Dentistry and Pharmaceutical Sciences, Okayama University, Okayama, Japan; ${ }^{8}$ Research Support Department, Drug Safety Research Laboratories, Pharmacokinetics and Bioanalysis Center, Shin Nippon Biomedical Laboratories Ltd, Kagoshima, Japan; ${ }^{9}$ Department of Hospital Pharmacy, Okayama University Hospital, Okayama, Japan.
\end{abstract}

Received December 26, 2017; Revised, February 8, 2018; Accepted, February 10, 2018; Published, February 13, 2018.

ABSTRACT - Purpose. Although the 2016 Japanese guidelines for the management of sepsis recommend de-escalation of treatment after identification of the causative pathogen, adherence to this practice remain unknown. The objective of this study was to evaluate the benefits of de-escalating treatment for sepsis patients at an advanced critical care and emergency medical centre. Methods. Based on electronic patient information, 85 patients who were transported to the centre by ambulance, and diagnosed with sepsis between January 2008 and September 2013 were enrolled and evaluated. Patients were divided into two groups with and without deescalation, and comparisons were conducted for several variables, including length of hospital stay, and length of antibiotic administration. Two types of subgroup analysis were conducted between patients with septic shock or positive blood cultures. Statistical analysis was conducted using chi-square and Mann-Whitney U tests. Results. The length of hospital stay after diagnosis was significantly shorter for the de-escalation group than for the non-de-escalation group. In the subgroup analysis, de-escalation for blood culture-positive patients was beneficial in terms of the length of hospital stay and length of antibiotic administration. Conclusions. The findings of this study suggest that sepsis treatment de-escalation is beneficial for treatment efficacy and appropriate use of antibiotics.

This article is open to POST-PUBLICATION REVIEW. Registered readers (see "For Readers") may comment by clicking on ABSTRACT on the issue's contents page.

\section{INTRODUCTION}

Sepsis is a life-threatening organ dysfunction caused by a dysregulated host response to infection (1). The reported mortality rates for sepsis are $25-30 \%$ and $40-70 \%$ for septic shock (2). Improving the survival rates for severe sepsis and septic shock requires not only treatment of the infection but also intensive care of the organ dysfunction and associated pathologies that occur successively. The treatment guidelines for sepsis are stipulated in the international guidelines for the management of sepsis (3), with descriptions of antimicrobial therapy, surgical management, and combined therapy.

During the treatment of sepsis, lower mortality rates have been associated with shorter periods between the recognition of the pathology and administration of antibiotics; in particular, a significantly lower mortality rate has been reported when antibiotics were administered within 1 hour of diagnosis (4). Therefore, swift administration of antibiotics based on empiric treatment is crucial following diagnosis (5). However, excessive use of antibiotics has been associated with the appearance of resistant bacteria and risk of destroying the patient's normal bacterial flora (6). Therefore, after

\footnotetext{
Corresponding author: Yoshito Zamami, Department of Clinical Pharmacology and Therapeutics, Tokushima University Graduate School of Biomedical Sciences, 3-18-15 Kuramoto, Tokushima 770-8503, Japan; e-mail: zamami@tokushimau.ac.jp
} 
administering empiric treatment, identification of the causative pathogen and, when the drug sensitivity is known, de-escalation to more narrowspectrum antibiotics is recommended. The antimicrobial de-escalation is definded as change to a narrow spectrum antibacterial drug or reduce the type of antimicrobial drug (7). The successful use of antibiotics in de-escalation has indicated that such use does not increase the recurrence or mortality rates for sepsis $(8,9)$, which results in improvements in survival rates and length of hospital stay in cases of ventilator-associated pneumonia (7). Further, deescalation has also been reported as a safe strategy even for patients with severe sepsis and septic shock admitted to the intensive care unit (ICU) (10).

A number of clinical studies regarding deescalation for patients with bacteraemia or traumatic injury have been undertaken (11-15). However, the outcomes of de-escalation of sepsis patients in the emergency domain have not been examined. Therefore, this study aimed to investigate the benefits of de-escalation specific to sepsis in the emergency domain, by retrospectively studying sepsis treatment at the advanced critical care and emergency medical centre (hereafter, emergency intensive care unit [EICU]) and evaluating the deescalation rates and suitability of empiric treatment.

\section{METHODS}

\section{Patients and data}

Information was collected from electronic patient medical record terminals at the hospital pharmacy. Patients who were diagnosed with sepsis and received treatment at the EICU between January 2008 and September 2013 were eligible. Patients aged $<18$ years or who did not receive proactive treatment were excluded. In addition, patients who were de-escalated after being transferred from the EICU to other hospital wards were excluded from the comparison of outcomes by de-escalation status.

Variables included sex, age, height, weight, body mass index (BMI), the condition for which patients were transported to the hospital and any complications, lactic acid levels, Acute Physiology and Chronic Health Evaluation (APACHE II) score (16), and Sequential Organ Failure Assessment (SOFA) score (17). Items used to distinguish between empiric treatment and de-escalation were the date and time of sepsis diagnosis, date of identification of the causative pathogen, antibiotics used, drug sensitivity of the causative pathogen, and the presence of neutropenia (data not shown). Outcomes included the length between the diagnosis of sepsis and discharge from the EICU (days), patient outcome (mortality rate) prior to discharge or transfer, and length of antibiotic administration (days).

Empiric treatment by doctors was defined as administration of suitable antibiotics during the period between the diagnosis of sepsis and identification of the causative pathogen. Deescalated patients were those for whom suitable empiric treatment was conducted and who met the following three criteria: clinical improvement in condition, identified causative pathogen receptive to more narrow-spectrum antibiotics, and no sustained neutropenia $\left(<1000 / \mathrm{mm}^{3}\right)$ or other serious immunodeficiency.

De-escalation was carried out based on the identification of the bacteria. A flow diagram on the timeline, from transportation to the emergency room to de-escalation, is presented in supplementary figure 1. In this study, de-escalation was defined as a change to more narrow-spectrum antibiotics or a reduction in the types of antibiotics used after identification of the causative pathogen and its drug sensitivity. Criteria for de-escalation followed those reported by Kollef et al. (9). Evaluation of the utility of de-escalation was conducted by dividing the subjects into two groups based on de-escalation and comparing and assessing the mortality rate, length of hospital stay (days), and length of antibiotic administration (days) between the two groups.

\section{STATISTICAL ANALYSIS}

The Student's t test, Welch's t test, or Mann-Whitney $\mathrm{U}$ test was used for comparing continuous variables, as appropriate. To compare categorical variables, the chi-square test was performed.

Comparisons were only conducted for those with available data for lactic acid levels, APACHE II score, and SOFA score. Two types of sub-group analysis were conducted, where the patients were further classified into those with septic shock, or those with positive blood culture only, to compare the influence on de-escalation outcome. $P<0.05$ was considered significant.

\section{Ethical considerations in research}

The survey content and methods to protect personal information were approved by the Okayama University Hospital Ethics Committee and in accordance with the stipulations on the handling of patient personal information (Ethics Committee Registration Number: 969) 


\section{RESULTS}

\section{Rate of de-escalation}

Empiric treatment was conducted for all 85 patients with sepsis, and de-escalation was considered suitable for 60 patients (Fig. 1). De-escalation was conducted for $21(35.0 \%)$ of these patients.

\section{Characteristics of de-escalation and non-de- escalation groups}

Patient background by de-escalation and non-deescalation status is provided in Table 1. Median age, sex distribution, median BMI, median lactic acid level, mean APACHE II score, median SOFA score, and the median time (in days) from the first blood collection and the identification of the microbes was not significantly different between the groups. The patients' complaints at the time of transportation are presented in supplementary Table 1 . The non-deescalation group contained more patients with liver failure and renal failure, compared to that of the deescalation group. The number of users taking antibiotics before and after de-escalation is shown in Supplementary Table 2. As a result of the deescalation, the relative proportion of carbapenem antibiotics that were used (such as Imipenem/Cilastatin and Piperacillin/Tazobactam) decreased, while the relative proportion of betalactam antibiotic usage increased.

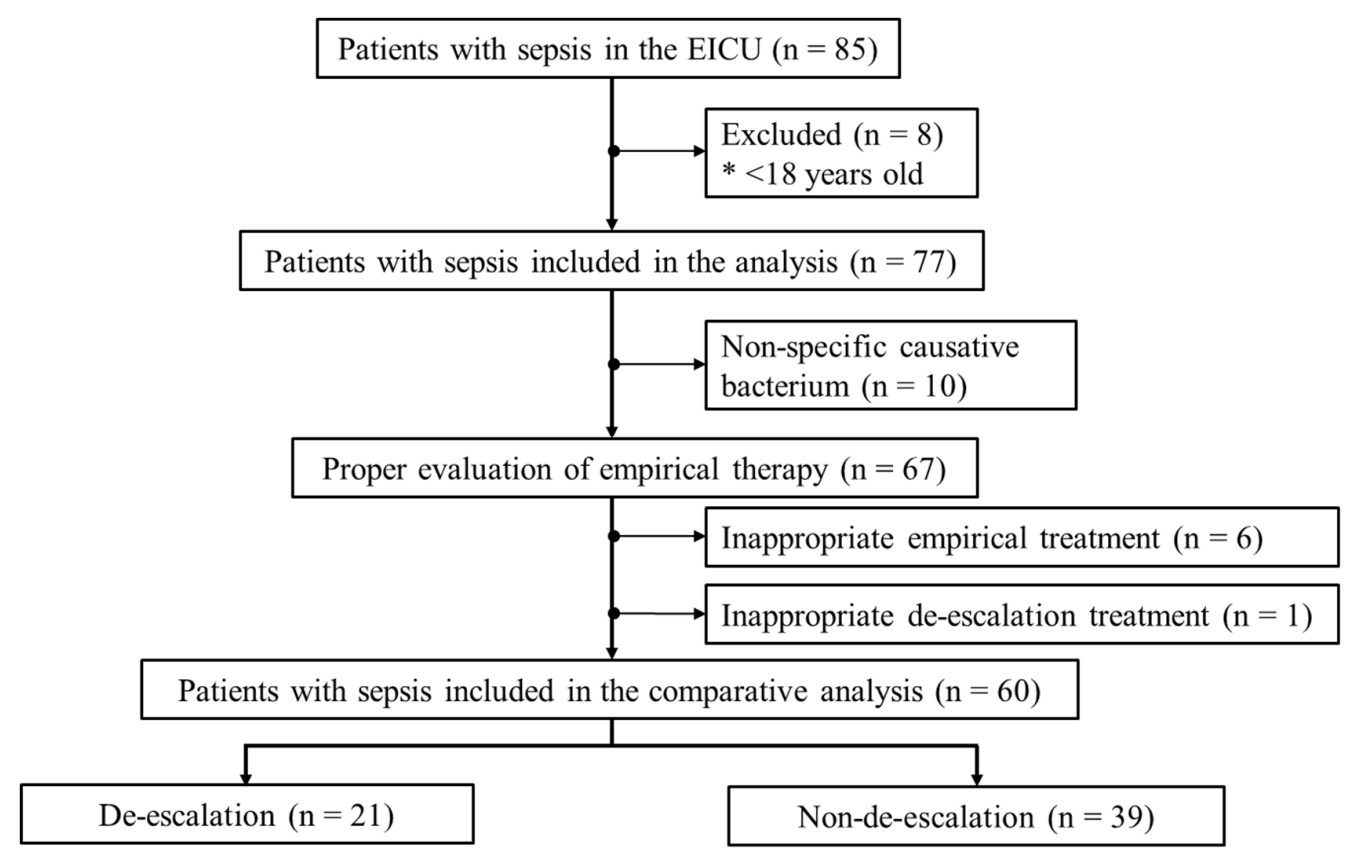

Figure 1. De-escalation for patients with sepsis

Table 1. Patient characteristics by de-escalation status

Values are reported as median (range), mean \pm standard deviation, or $\mathrm{n}$.

\begin{tabular}{|c|c|c|c|}
\hline & De-escalation group & Non-de-escalation group & p value \\
\hline Age (years) & $61.1(23-89)$ & $69.0(20-89)$ & 0.053 \\
\hline \multicolumn{4}{|l|}{ Sex } \\
\hline Male & 13 & 29 & 0.315 \\
\hline Female & 8 & 10 & \\
\hline BMI (kg/m²) & $20.9(10.74-30.42)$ & $21.0(10.65-60.35)$ & 0.11 \\
\hline Lactic acid level (mmol/L) & $1.9(0.7-7.1)$ & $2.3(0.7-19.0)$ & 0.27 \\
\hline APACHE II score & $17.5 \pm 5.0$ & $19.0 \pm 8.1$ & 0.485 \\
\hline SOFA score & $6.5(2-13)$ & $7.5(2-15)$ & 0.936 \\
\hline $\begin{array}{l}\text { Median days before } \\
\text { identification of the microbes }\end{array}$ & $4(2-9)$ & $3(2-8)$ & 0.187 \\
\hline
\end{tabular}

Comparison of outcomes by de-escalation and non-de-escalation groups 
Mortality rate and length of antibiotic administration were not significantly different between the two groups, although they were both higher in the nonde-escalation group (Table 2).

The median length of hospital stay was significantly longer in the non-de-escalation group (26 days) than in the de-escalation group (12 days; $p$ $=0.028$ ).

\section{Subgroup analysis}

In the group of patients with septic shock, the rate of de-escalation was $33.3 \%(10 / 30)$. Mortality rate, length of hospital stay, and length of antibiotic administration did not differ by de-escalation status (Table 3).

In the group of patients with only positive blood cultures, the rate of de-escalation was 39.0\% (16/41). Mortality rate, length of hospital stay, and length of antibiotic administration were all superior for the deescalation group. The differences in the length of hospital stay and length of antibiotic administration were significant (Table 4).

Table 2. Comparison of outcomes by de-escalation status

\begin{tabular}{|c|c|c|c|}
\hline & De-escalation group $(n=21)$ & Non-de-escalation group $(n=39)$ & p value \\
\hline $\begin{array}{l}\text { Mortality rate } \\
\text { (Death/Survival) }\end{array}$ & $\begin{array}{l}9.5 \% \\
(2 / 19)\end{array}$ & $\begin{array}{l}23.1 \% \\
(9 / 30)\end{array}$ & 0.227 \\
\hline $\begin{array}{l}\text { Length of hospital stay } \\
\text { (days) }\end{array}$ & $12(4-138)$ & $26(5-204)$ & 0.028 \\
\hline Length of antibiotic therapy (days) & $12(4-34)$ & $16(5-71)$ & 0.071 \\
\hline
\end{tabular}

Table 3. Influence of de-escalation in patients with septic shock

\begin{tabular}{lllc}
\hline & De-escalation group $(\mathbf{n}=\mathbf{1 0})$ & Non-de-escalation group $(\mathbf{n}=\mathbf{2 0})$ & $\mathbf{p}$ value \\
\hline Mortality rate & $10.0 \%$ & $40.0 \%$ & 0.204 \\
(Death/Survival) & $(1 / 9)$ & $(8 / 12)$ & 0.184 \\
Length of hospital stay (days) & $11(7-51)$ & $21(5-81)$ & 0.26 \\
\hline Length of antibiotic therapy (days) & $10(6-21)$ & $15(5-70)$ &
\end{tabular}

Values are reported as median (range) or a percentage.

Table 4. Influence of de-escalation in blood culture-positive patients

\begin{tabular}{llll}
\hline & De-escalation group $(\mathbf{n}=\mathbf{1 6})$ & Non-de-escalation group $(\mathbf{n}=\mathbf{2 5})$ & $\boldsymbol{p}$ value \\
\hline Mortality rate & $12.50 \%$ & $24.0 \%$ & 0.448 \\
(Death/Survival) & $(2 / 14)$ & $(6 / 19)$ & \\
Length of hospital stay (days) & $11(4-138)$ & $26(5-204)$ & 0.030 \\
Length of antibiotic therapy (days) & $12(4-34)$ & $16(5-71)$ & 0.041 \\
\hline
\end{tabular}

Values are reported as median (range) or a percentage.

\section{DISCUSSION}

The percent of de-escalation in the treatment of patients with sepsis in the EICU was $35.0 \%(21 / 60)$, similar to the results of previous studies conducted (11-14). Reasons not to de-escalate may have included difficulty controlling antimicrobial treatment of sepsis.

Regarding the benefits of de-escalation, the length of hospital stay was significantly shorter for the de-escalation group than for the non-deescalation group. Because the reason for discharge from the EICU was improved symptoms, the results indicate that those with the swiftest improvement in symptoms had the shortest hospital stays; in other words, the severity of the patient's condition may have biased the results. However, the significant difference between the de-escalation and non-deescalation groups despite no bias in severity indicates that de-escalation contributed to the shorter 
hospital stay. Therefore, treatment de-escalation might be beneficial for patients with sepsis.

The length of antibiotic administration is important for evaluating the appropriate use of antibiotics. Long-term antibiotic administration prolongs hospital stays and treatment duration and adversely affects prognosis for some patients owing to side effects such as microbial substitution due to resistant bacteria or the resistant bacteria themselves. As such, the duration of antibiotic administration for bacteraemia and other conditions is generally limited to 14 days (18). The median length of antibiotic administration in the present study was 12 days for the de-escalation group, compared with 16 days for the non-de-escalation group, indicating a tendency for de-escalation to help shorten the length of antibiotic administration.

Given that shorter antibiotic administration may be correlated with the duration of hospital stay, a subgroup analysis was conducted between patients with septic shock or who were blood culture positive, which are considered to have a major influence on the decision for de-escalation. The selection of antibiotics depends on whether there is sufficient time to reflect the results of blood culture tests. In the present study, no significant difference was observed in the length of hospital stay or antibiotic administration based on de-escalation status for patients with septic shock. However, for blood culture-positive patients, the length of hospital stay and length of antibiotic administration were significantly shorter for the de-escalation group. The guidelines recommend drawing blood cultures prior to commencing antibiotics in all cases of sepsis. ${ }^{3}$ Further, the aseptic collection of sample specimens from the possible infection source site for smear tests and culture identification and sensitivity tests is also recommended (19-21). The results of the present study may provide important evidence to substantiate these recommendations for blood cultures. However, for patients with septic shock, there is almost no leeway in clinical practice to delay antibiotic administration for the purpose of drawing blood cultures. We believe that this affected the outcomes between the two subgroups.

The results of the present study indicate that the selection of the antibiotic agent and de-escalation on the basis of blood culture tests are beneficial for treatment outcomes of sepsis in the emergency domain. Therefore, further investigation of the utility of blood culture testing is warranted.

\section{CONFLICTS OF INTEREST}

The authors have indicated that they have no conflicts of interest regarding the content of this article.

\section{ACKNOWLEDGEMENTS}

We thank a native English speaker for checking the language and grammar used in this manuscript (Editage, Tokyo, Japan).

\section{REFERENCES}

1. Singer M, Deutschman CS, Seymour CW, ShankarHari M, Annane D, Bauer M, et al. The Third International Consensus Definitions for Sepsis and Septic Shock (Sepsis-3). JAMA. 2016 Feb 23;315(8):801-10. doi: 10.1001/jama.2016.0287.

2. Russell JA. Management of sepsis. N Engl J Med. 2006 Oct 19;355(16):1699-713.

3. Rhodes A, Evans LE, Alhazzani W, Levy MM, Antonelli M, Ferrer R, et al. Surviving Sepsis Campaign: International Guidelines for Management of Sepsis and Septic Shock: 2016. Intensive Care Med. 2017 Mar;43(3):304-377. doi: 10.1007/s00134017-4683-6.

4. Gaieski DF, Mikkelsen ME, Band RA, Pines JM, Massone R, Furia FF, et al. Impact of time to antibiotics on survival in patients with severe sepsis or septic shock in whom early goal-directed therapy was initiated in the emergency department. Crit Care Med. 2010 Apr;38(4):1045-53. doi: 10.1097/CCM.0b013e3181cc4824.

5. Lee CC, Lee $\mathrm{CH}$, Chuang MC, Hong MY, Hsu HC, Ko WC. Impact of inappropriate empirical antibiotic therapy on outcome of bacteremic adults visiting the ED. Am J Emerg Med. 2012 Oct;30(8):1447-56. doi: 10.1016/j.ajem.2011.11.010.

6. Falagas ME, Kopterides P. Risk factors for the isolation of multi-drug-resistant Acinetobacter baumannii and Pseudomonas aeruginosa: a systematic review of the literature. J Hosp Infect. 2006 Sep;64(1):7-15.

7. Kollef MH, Morrow LE, Niederman MS, Leeper KV, Anzueto A, Benz-Scott L, et al. Clinical characteristics and treatment patterns among patients with ventilator-associated pneumonia. Chest. 2006 May;129(5):1210-8.

8. Shime N, Satake S, Fujita N. De-escalation of antimicrobials in the treatment of bacteraemia due to antibiotic-sensitive pathogens in immunocompetent patients. Infection. 2011 Aug;39(4):319-25. doi: 10.1007/s15010-011-0116-6.

9. Morel J, Casoetto J, Jospé R, Aubert G, Terrana R, 
Dumont A, et al. De-escalation as part of a global strategy of empiric antibiotherapy management. A retrospective study in a medico-surgical intensive care unit. Crit Care. 2010;14(6):R225. doi: 10.1186/cc9373.

10. Garnacho-Montero J, Gutiérrez-Pizarraya A, Escoresca-Ortega A, Corcia-Palomo Y, FernándezDelgado E, Herrera-Melero I, et al. De-escalation of empirical therapy is associated with lower mortality in patients with severe sepsis and septic shock. Intensive Care Med. 2014 Jan;40(1):32-40. doi: 10.1007/s00134-013-3077-7.

11. Shime N. Antimicrobial therapy for bacteremia: is the de-escalation strategy performed appropriately? J Jpn Soc Surg Infect. 2006 Nov:3(4):495-498.

12. Muro T, Miyoshi K, Umeda $\mathrm{Y}$, Takemoto S, Nakamura K, Kamimura H, et al. The effects and problems of educational intervention by an infection control team aimed at tapering off carbapenem. J Jpn Soc Hosp Pharm. 2009 Nov:45(11):1521-1524.

13. Shirai K, Toyoda I, Murakami N, Yoshida S, Kato H, Doi $\mathrm{T}$, et al. Comparison of the antibiotics administration tactics before and after intervention of the ICT in trauma patients. J Jpn Soc Emergency Med. 2010 Jun;13(3):334-340.

14. Kudo S, Tandai K, Gamazaw K. Evaluation of a monitoring strategy for promotion of dose deescalation of carbapenems without using a notification and permission system. J Jpn Soc Hosp Pharm. 2012 Aug;48(8):981-985.

15. Tanaka H, Taguchi K, Hirabayashi M, Namiki M, Ugagin K, Marumo K, et al. Economical benefit of antimicrobial chemotherapy with a tapering schedule in blood culture-positive patients. J Jpn Soc Hosp Pharm. 2011 Mar;47(3):301-303.

16. Knaus WA, Draper EA, Wagner DP, Zimmerman JE. APACHE II: a severity of disease classification system. Crit Care Med. 1985 Oct;13(10):818-29.

17. Vincent JL, Moreno R, Takala J, Willatts S, De Mendonça A, Bruining H, et al. The SOFA (Sepsisrelated Organ Failure Assessment) score to describe organ dysfunction/failure. On behalf of the Working Group on Sepsis-Related Problems of the European Society of Intensive Care Medicine. Intensive Care Med. 1996 Jul;22(7):707-10.

18. Fagon JY, Chastre J, Wolff M, Gervais C, ParerAubas S, Stéphan F, et al. Invasive and noninvasive strategies for management of suspected ventilatorassociated pneumonia. A randomized trial. Ann Intern Med. 2000 Apr 18;132(8):621-30.

19. Canadian Critical Care Trials Group. A randomized trial of diagnostic techniques for ventilatorassociated pneumonia. N Engl J Med. 2006 Dec 21;355(25):2619-30.
20. Berton DC, Kalil AC, Teixeira PJ. Quantitative versus qualitative cultures of respiratory secretions for clinical outcomes in patients with ventilatorassociated pneumonia. Cochrane Database Syst Rev. 2014 Oct 30;(10):CD006482. doi: 10.1002/14651858.CD006482.pub4.

21. Mermel LA, Allon M, Bouza E, Craven DE, Flynn P, O'Grady NP, et al. Clinical practice guidelines for the diagnosis and management of intravascular catheterrelated infection: 2009 Update by the Infectious Diseases Society of America. Clin Infect Dis. 2009 Jul 1;49(1):1-45. doi: 10.1086/599376. 